\title{
Neurofibromatose de type 1 ou maladie de von Recklinghausen : de nombreuses questions restent posées
}

Ia neurofibromatose de type 1 (NFI) est une des maladies héréditaires les plus fiéquentes. Elle a été décrite la première fois en 1793, puis mieux documentée en 1882 par von Recklinghausen, d'où son appellation de maladie de von Recklinghausen ou neurofibromatose de type 1 [1]. Elle fait partic des phacomatoses, en référence aux taches cutanées, un des signes fréquemment rencontrés dans cette maladie. La NFl se distingue des autres phacomatoses par le type de lésions primaires responsables de la symptomatologie associée au syndrome. En effet, les cellules impliquées dans le développement de la NFI sont en grande partie dérivées des cellules do la crête neurale, ce qui n'est pas aussi systématique pour les autres maladies de type phacomatose. On peut aussi trouver des anomalies de développement de cellules provenant de l'ectoderme, du neurectoderme, du mésoderme et de l'endoderme.

\section{Une grande hétérogénéité clinique}

I a maladie de von Recklinghausen représente une entité cliniquement hétérogène caractérisée par la présence de malformations tumorales souvent bénignes dans les organes d'origine ectodermique, principalement la peau et le système nerveux mais aussi des manifestations osseuses, vasculaires et viscérales. Bien que peu fréquentes, des difficultés d'apprentissage et de concentration chez certains enfants peuvent se rencontrer. Cependant, ces difficultés sont inconstantes et le plus souvent très modérées [2].

Les signes cutanés sont les plus constants et les plus précoces. Parmi ceux-ci les taches café au lait sont présentes dans environ $99 \%$ des cas. Des $m / s 1^{\circ} 1$, vol. 11 , janvier 95 tumeurs cutanées, généralement bénignes, de type neurofibrome sont aussi caractéristiques du syndrome. Ceux-ci peuvent être très importants et portent alors le nom de névromes plexiformes en nappe ou en plaque. Des leucémies myéloïdes chroniques sont aussi observées. Environ 10 \% des enfants avec des syndromes myéloprolifératifs sont atteints de NFl.

Différentes manifestations neurologiques permettent de distinguer deux formes de la maladie. Celles-ci sont rarement associées chez un même individu, mais peuvent, en revanche, se rencontrer dans une même famille. I'une des formes est caractérisée par de multiples neurofibromes et des neurinomes. Bien que le phéochromocytome soit le plus souvent une tumeur bénigne, des phéochromocy-

Tableau I

\section{CRITĖRES DE DIAGNOSTIC CLINIQUE DE NF1}

1. Au moins 5 taches café au lait $>$ à $1,5 \mathrm{~cm}$ après la puberté $>$ à $0,5 \mathrm{~cm}$ avant la puberté

2. Au moins deux neurofibromes quel que soit le type

3. Au moins un neurofibrome plexiforme

4. Gliome du nerf optique

5. Au moins 2 nodules de Lisch

6 . Lésions osseuses

7. Un apparenté de premier degré atteint de NF1

Pour faire le diagnostic de la neurofibromatose de type $\mathrm{l}$, au moins deux de ces signes doivent être réunis chez un même individu. tomes malins et des neurofibrosarcomes peuvent être observés. L'autre forme est de pronostic plus sévère, parfois associée à des gliomes du nerf optique, des méningiomes et des neurinomes. Le neurinome de l'acoustique constitue une forme distincte de neurofibromatose puisqu'il est associé uniquement à la neurofibromatose de type 2. D'autres signes peuvent être observés, comme les anomalies ophtalmiques de type phacome de la rétine, le névrome plexiforme de la paupière supéricure, mais surtout les hamartomes pigmentés de l'iris ou nodules de I isch qui constituent un bon outil diagnostique, étant présents che\% environ $94 \%$ des malades âgés de plus de 6 ans. Les manifestations osseuses se retrouvent dans $50 \%$ des cas, et regroupent des pseudarthroses, des cypho-scolioses et des hypertrophies osseuses. I es manifestations viscérales sont dominées par les neurinomes médiastinaux et les tumeurs digestives et viscérales. Plusieurs de ces signes peuvent se retrouver chey un même patient.

La NFI est une maladie très hétérogène avec des formes sévères et des formes frustes limitées à quelques taches café au lait et des nodules de I isch. L'hétérogénéité clinique et les différences d'âge d'apparition des symptômes associés à la NF1 rendent parfois le diagnostic difficile. Les critères de diagnostic en vigueur sont décrits dans le Tablean I. Riccardi estime à $25 \%$ la fréquence des formes graves avec environ $5 \%$ à $10 \%$ de tumeurs du système nerveux central, $2 \%$ à $5 \%$ de retard mental important et $5 \%$ à 8 $\%$ de dégénérescence maligne. La très grande variabilité de la maladie se manifeste aussi bien à l'intérieur des familles qu'entre les différentes fa- 
milles. Ainsi, l'existence d'une forme bénigne chez plusieurs sujets n'exclut pas la possibilité qu'apparaisse une forme grave dans la famille.

L'évolution naturelle de la maladie est actuellement totalement imprévisible. Malgré le clonage du gène, aucune corrélation génotype/phénotype n'a encore été établie.

\section{Une transmission autosomique dominante}

La NFl se transmet selon un mode autosomique dominant. Elle touche toutes les races avec une incidence de 1 pour 3500 naissances [2]. Environ $50 \%$ des cas sont sporadiques, liés à une fréquence élevée de nouvelles mutations, le plus souvent d'origine paternelle (22 cas sur 24) (m/s $n^{\circ} 4$, vol. 6, p. 397) [3]. Cependant, aucun effet de l'âge paternel dans l'apparition des néo-mutations n'a été démontré. Le taux de néo-mutation a été estimé à $10^{-4}$ par gamète et par génération. Les symptômes semblent se développer plus précocement et de manière plus grave lorsque la mutation est transmise par la mère. Peu de délétions ont été rapportées, contrairement à ce qui a été décrit dans des maladies génétiques associées à un gène de grande taille. Par exemple, on dénombre $60 \%$ de délétions parmi les mutations du gène de la dystrophine. Bien que rares, des mosaïques germinales et somatiques ont été caractérisées. Les mutations à l'origine des mosaïques somatiques peuvent survenir très précocement, et, de ce fait, impliquer de nombreux tissus, entraînant de multiples manifestations cliniques [4].

\section{Un grand gène}

Le gène responsable de la maladie a été localisé en 1987 sur le chromosome 17 grâce à la description de deux translocations équilibrées impliquant la bande $17 \mathrm{q} 11.2$ et à des études de liaison avec des marqueurs de ce même chromosome [5]. Par la suite, la caractérisation plus précise des deux points de cassures des translocations associées à une NFl a montré qu'ils se trouvaient distants l'un de l'autre de $60 \mathrm{~kb}$. L'approche par clo- nage positionnel a alors pu être envisagée.

En 1990, les groupes de Collins et White ont isolé l'ADNc du gène $N F 1$ $\left(m / s n^{\circ} 8\right.$, vol. $6, p$. 815) $\left.[6,7]\right)$. Le gène s'étend sur plus de $300 \mathrm{~kb}$, contient 52 exons et présente la particularité de contenir, à l'intérieur de l'intron 27, trois gènes transcrits en direction opposée (figure 1) : le gène codant pour l'oligodendrocyte myelin glycoprotein $(O M G P)$, un peptide de surface du système nerveux central, et deux gènes homologues d'oncogènes murins sans fonction définie chez l'homme (EVI2A, EVI2B), qui codent pour des protéines transmembranaires. Il n'existe aujourd'hui aucun argument permettant d'impliquer un de ces gènes dans les manifestations cliniques de la NFl (figure 1). Une autre séquence correspondant à un pseudogène de l'adénylate kinase (AK3) a été retrouvée dans l'intron 39. La taille du messager du gène $N F 1$ est de 13 $\mathrm{kb}$. La phase ouverte de lecture est de 8454 nucléotides. Cet ARN code pour une protéine de 2818 acides aminés appelée neurofibromine $\left(\mathrm{m} / \mathrm{s} n^{\circ} \mathrm{l}\right.$, vol. 8, p. 91).

Il existe au niveau des exons $23 \mathrm{a}$ et 48 a des sites d'épissage alternatif qui engendrent plusieurs isoformes de la neurofibromine. Le premier correspond à une insertion de 63 nucléotides, le second à une insertion de 54 nucléotides. Cette dernière forme est fortement exprimée dans les muscles lisses, cardiaque et squelettique. On comnaît peu d'anomalies cardiaques associées à la NFl, cependant, bien qu'un petit nombre de cellules de la crête neurale contribue au développement et à la différenciation du cœur, différentes expériences montrent, chez la souris, que l'absence d'expression du gène $N F 1$ entraîne la mort de l'embryon à 13 jours, avec des œdèmes généralisés, due à des anomalies cardiaques (voir les modèles animaux) [8]. Ces anomalies résultent de l'absence de cellules de la crête neurale. Malgré ces premières données, la signification fonctionnelle de ces différentes isoformes reste inconnue. Elles sont conservées au cours de

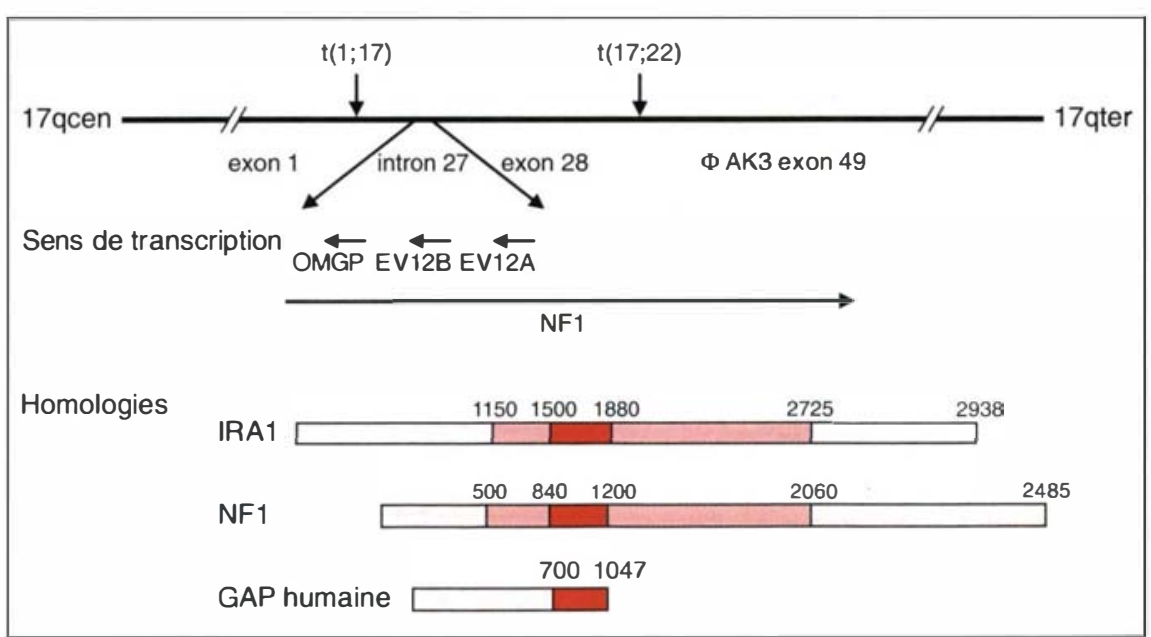

Figure 1. Cartographie du gène NF1. Les deux translocations qui ont permis d'isoler le gène sont notées dans la partie supérieure du schéma. Les sens de transcription des gènes OMGP, EVI2B, EVI2A contenus dans l'intron 27 et du gène NF1 sont donnés dans la partie intermédiaire. Les analogies de la protéine codée par NF1 avec les séquences protéiques IRA1 de levure et GAP humain sont décrites dans la partie basse du schéma. Les numéros des acides aminés sont notés au-dessus de chaque protéine. La boîte rouge correspond à la région des 360 acides aminés conservés dans les trois séquences, les boîtes roses correspondent à la région de forte similitude entre NF1 et IRA. 


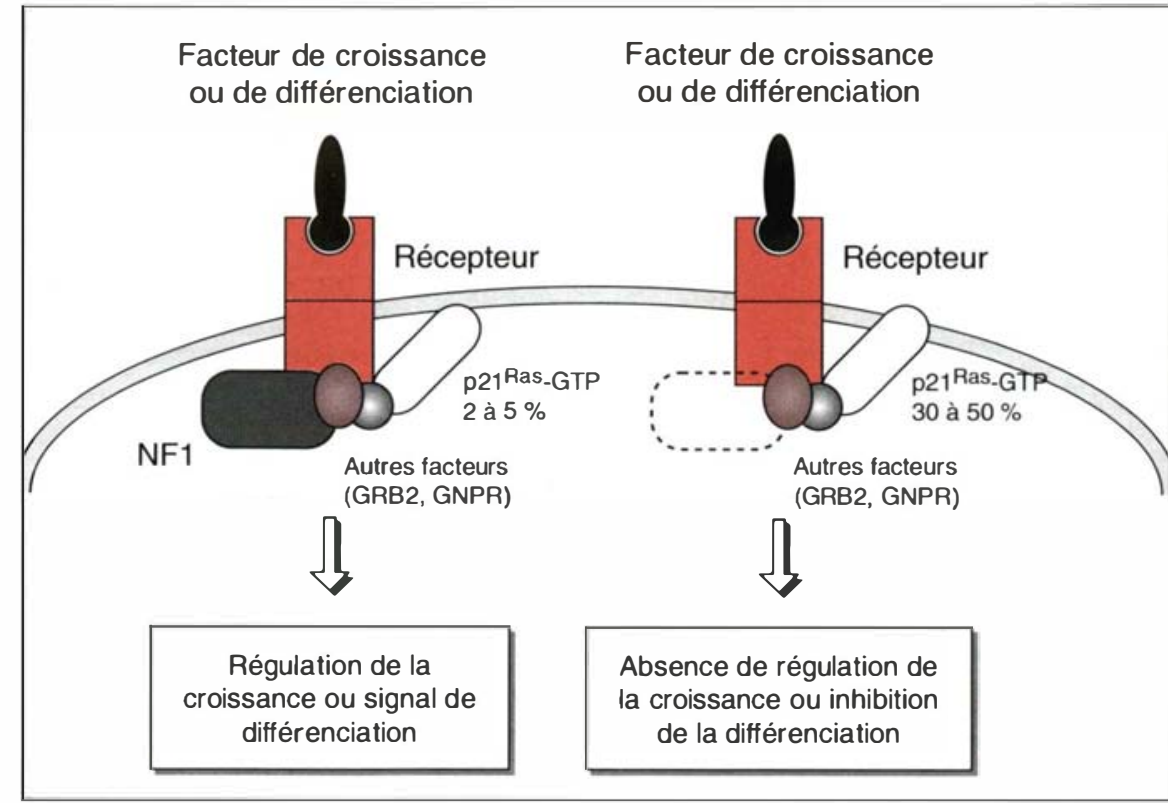

Figure 2. Mode d'action possible de la neurofibromine. A gauche, la neurofibromine catalyse l'hydrolyse du GTP lié à p21Ras, la majorité de cette protéine se trouvant sous sa forme inactive liée au GDP. Ainsi, la neurofibromine pourrait contrôler négativement la croissance et éventuellement relayer un signal de différenciation. A droite, en l'absence d'activité suffisante de la neurofibromine, une grande partie de $p 21^{\text {Ras }}$ est sous sa forme activée, liée au GTP, ce qui aboutit à une stimulation continue de la croissance. De plus, un signal de différenciation pourrait être perdu. GRB2 et GNPR sont respectivement un adaptateur et un facteur d'échange du GDP pour le GTP. (D’après [11].)

l'évolution, laissant penser qu'elles jouent un rôle bien défini dans la fonction de la neurofibromine. Toutes isoformes confondues, l'expression de la neurofibromine est ubiquitaire. D'autres études sur l'expression des différentes formes de la neurofibromine dans les différents tissus dérivés des cellules de la crête neurale devraient apporter plus d'information sur le rôle de ces différentes isoformes.

\section{Une protéine : plusieurs fonctions?}

Les analyses de séquence ont permis de reconnaître une région conservée de 360 résidus, dont 15 acides aminés, répartis en trois blocs, sont strictement conservés [9]. Ce domaine est similaire au domaine catalytique de type RasGTPase des protéines p $120^{\text {GAP }}$ des mammifères mais surtout de Iralp, Ira2p et Sarl des levures Saccharomyces cerevisiae et Schizosaccharomyces pombe, ainsi que de GAPl de dro$\mathrm{m} / \mathrm{s} n^{\circ} \mathrm{l}$, vol. 11 , janvier 95 au choc thermique. Les mutations du domaine GAP de la neurofibromine peuvent avoir différents effets: (1) la disparition totale de la fonction NFIGRD (NFl GAP related domain) ; (2) la réduction de son activité ; (3) une conservation de la fonction. L'absence de complémentation lorsque le domaine GAP est muté, ainsi que la complémentation complète par le gène normal, démontrent le rôle majeur de la neurofibromine dans le contrôle négatif de $\mathrm{p} 21^{\text {Ras }}$, cible des protéines IRA.

L'absence de neurofibromine dans une cellule inhiberait donc la voie de différenciation contrôlée par p21 Ras et pourrait stimuler la voie mitogène en ne réprimant plus le rôle activateur de croissance de $\mathrm{p} 2 \mathrm{l}^{\text {Ras }}$. L'homologie fonctionnelle entre les protéines NFl, GAP et IRA suggère deux voies possibles pour expliquer la physiopathologie moléculaire de la NFI [11] (figure 2): (1) la fonction normale de la neurofibromine serait de contrôler négativement la transmission d'un signal de croissance par $\mathrm{p} 21^{\mathrm{Ras}}$; (2) la neurofibromine serait un effecteur de la transmission du signal de différenciation par p21 Ras. Dans les deux cas, l'absence de neurofibromine serait responsable d'une croissance anormale des cellules.

Cependant, la neurofibromine possède peut-être d'autres domaines fonctionnels non encore identifiés situés plus en avant dans la région C-terminale de la protéine, comme le suggère la rareté des mutations dans le domaine GRD.

tion du taux de p21 Ras-GTP et, de ce fait, augmente l'activité de $\mathrm{p} 21^{\text {Ras }}$ connue pour intervenir dans la transduction des signaux contrôlant la croissance et la différenciation cellulaire.

De nombreuses expériences ont permis de démontrer que la neurofibromine est impliquée dans le contrôle de la différenciation et de la prolifération cellulaire en réglant l'activité du proto-oncogène p2lRAS [10]. Parmi celles-ci, certaines ont consisté à essayer de complémenter la sensibilité au choc thermique des levures IRA; soit par la neurofibromine normale, soit par des mutants de la région GAP de la neurofibromine. La neurofibromine normale complémente les levure IRA qui redeviennent insensibles
Des mutations constitutionnelles difficiles à démasquer

Malgré la grande taille du gène, les recherches de remaniement se sont avérées peu fructueuses [12]. Peu de translocations et de délétions [13] ont jusqu'alors été décrites. Seules 54 mutations $(10 \%)$ ont été retrouvées pour plusieurs centaines de patients testés. Ces mutations ne touchent pas systématiquement le domaine GAP. L'exon 31 est le plus fréquemment touché sans pour cela définir un point chaud de mutations. Le promoteur du gène est encore mal connu, ainsi que la région 3' qui n'a pas encore été clonée. Des mutations dans ces régions 
pourraient entrainer des modifications d'expression du gène ou de la stabilité du messager. Une duplication en 3' du gène, responsable dans une famille d'une association de NFI et de syndromes de Noonan* el de Watson*, semblerait confirmer l'existence d'au moins un autre domaine fonctionnel [14]. Cette duplication de 42 pb siège dans l'exon 28 en position 17(01, à environ 500 pb du domaine (iAP de la protéine, et est très strictement associéc à ce phénotype. Elle est à l'origine de la synthèse d'une protéine anormale ; celle-ci pourrait expliquer l'association de différents syndromes caractéristiques dans certaines familles. C'est la seule association comnuc à ce jour entre un type de mutation et un phénotype. En effet, une étude portant sur 175 individus de 48 familles, incluant 6 paires de jumeaux monozygotes, a permis de démontrer que l'expression phénotypique de NFl due à différents types de mutation du gène est trop hétérogène et doit faire intervenir l'expression de gènes modificateurs, spécifiques d'un trait de la maladie, chaque trait pouvant apparaître indépendamment l'un de l'autre [15]. Pour les taches café au lait et les neurofibromes, la corrélation est plus forte chez les jumeaux monozygotes qu'entre apparentés de premier degré et baisse encore pour des parents plus éloignés. Cela suggère que, bien que les mutations du gène $N F 1$ jouent un rôle majeur dans l'expression de la maladie, le type de mutation joue un rôle mineur dans la variation de cette expression.

\section{Encore un gène suppresseur de tumeur}

Ice caractère prédisposant à l'apparition de tumeurs de la neurofibromatose de type 1 laissait supposer que le gène responsable de cette maladic était un gène suppresseur de tumeur. Dans cette hypothèse, des pertes d'allèles ont été recherchées sur le chromosome 17 dans les tumeurs associées ou non à une NF1, conformément à la théorie des deux événements de Kinudson [16]. Des pertes d'allèles de ce chromosome ont été retrouvées dans des tumeurs développées par des malades présentant une NF1, comme des neurofibrosarcomes, des phéochromocytomes et des schwamnomes pour les plus fréquentes $[17,18]$. Très souvent, cependant, celles-ci concernent la totalité du chromosome 17, voire uniquement le bras court (un cas de glioblastome), région comprenant le gène de la p53. I)es mutations ponctuelles de ce gène ont été décrites dans ces tumeurs, laissant donc supposer son implication dans le développement des tumeurs associées à la NFl. Une perte d'allèle limitée au gène NFl (délétion de $20(0)$ kb) a été décrite dans un neurofibrosarcome [19]. D'autres cas de perte d'allèle homozygote ont été décrits dans des cas de syndromes myélodysplasiques associés à une NF1, où l'allèle NF1 muté est conservé et l'allèle normal perdu dans la tumeur [20]. Ces exemples sont les seuls excmples de perte d'allèle homozygote dans une tumeur spécifiquement associée à une NFl qui permettent de soutenir l'hypothèse d'une fonction de suppresseur de tumeur pour ce gène. I'existence de mutations ponctuelles dans les tumeurs sans perte d'allèle ne peut en revanche être exclue, compte tenu de la difficulté de détection de ces mutations. Cependant, si le gène NFl est un gène suppresseur de tumeur, les mutations somatiques devraient être fréquentes, compte tenu du fort taux de néo-mutation caractéristique de la maladie. Des pertes d'allèles du chromosome 17 ont aussi été retrouvées dans d'autres tumeurs non associées à une NF1, comme les cancers coliques, des cancers du sein, des astrocytomes, des mélanomes, des neuroblastomes, pour les mieux documentés.

Bien que les pertes d'allèles du chromosome 17 soient rares dans les tumeurs associées à la NFl, l'idée d'un rôle suppresseur de tumeur du gène a été confortée par une expérience de fusion cellulaire aboutissant à l'introduction d'un chromosome 17 normal dans une lignée de neuroblastome. I a perte de tumorigénicité alors observée dans cette lignée a permis d'impliquer le chromosome 17 dans la perte du phénotype tumoral [21, 22]. Cette expérience ne permet cependant pas de discriminer le bras court du bras long et ainsi d'incriminer le gène NF1 luimême, plutôt que le gène de la p53.
Afin de mesurer le taux d'expression du gène NFl dans les tumeurs, la quantité d'ARN codant pour la neurofibromine a été contrôlée par Northerm blot dans dix lignées indépendantes de neuroblastomes. Quatre présentent un taux d'expression de la neurofibromine très diminué ou même nul. Parmi celles-ci, deux ont une mutation du gène NFl démontrant ainsi que des mutations du gène de la neurofibromine peuvent intervenir dans le développement des neuroblastomes, très rarement associés à une NF 1, mais aussi dans d'autres tumeurs rencontrées dans ce syndrome. Cependant, contrairement à ce qui pouvait être attendu, aucune augmentation du taux de $p 21^{\text {Ras }}$-GTP n'est enregistrée dans les neuroblastomes avec mutation du gène $N F 1$. Une des mutations caractérisée dans les neuroblastomes entraîne une baisse de la stabilité de l'ARNm qui voit sa durée de vie passer de 24 h a 3 h. En revanche, le dosage de l'activité $\mathrm{p} 21^{\text {Ras }}$ dans les tumeurs associées à une NF1 montre que $30 \%$ à 50) \% de la protéine est, sous sa forme active, liće au GTP, alors que dans des cellules NIH3T3 témoins, le pourcentage est de $2 \%$ à $5 \%[21,23]$. I e défaut d'activité de la neurofibromine n'est pas, dans ces cas, compensé par une augmentation de l'activité pl20(ap. Cela laisse penser que la neurofibromine contribue de manière différente à la régulation négative de p21 $1^{\text {Ras }}$ selon le type de cellule dans lequel elle s'exprime, et que des protéines comme pl20(6) on d'autres of fecteurs de $\mathrm{p} 21^{\text {Ras }}$ pourraient compenser son absence (figure 2). Aucun argument ne permet jusqu'alors d'expliquer ces différences observées dans la régulation très complexe de l'activité de p21 Ras.

I.e même type d'observation a été fait pour un autre gène suppresseur de tumeur, WT1 en 11 pl3, qui prédispose à l'apparition de néphroblastome. En effet, différentes équipes ont montré qu'un néphroblastome associé à une mutation constitutionnelle du gène WTl peut se développer malgré une expression résiduclle du gène WTI pouvant aller jusqu'à 50 \% du taux normal [24]. De plus, comme pour le gène de la neurofibromine, très peu de mutations (10 \%) sont détectées dans les tissus tumoraux, posant ainsi 
le probleme du mode d'action de ces protéines et du rôle potentiel des différentes isoformes puisque, jusqu'a présent, aucune étude n'a permis de déterminer le taux relatif de ces dernières dans les tumeurs.

Des expériences récentes, utilisant un polymorphisme Rsal exonique, ont permis de démontrer que dans des fibroblastes de 6 cas sur 8 de NFI on notait une différence dans les quantités de messagers transcrits à partir de chacun des deux allèles [25]. I e même type d'étude fait sur des cas familiaux a permis de déterminer que l'allèle le plus faiblement exprimé était celui porteur de la mutation. Ces différences d'expression sont indépendantes du parent transmetteur, excluant ainsi tout phénomène d'empreinte parentale qui aurait pu expliquer cette distorsion dans l'expression des deux allèles.

\section{Enfin des modèles animaux}

Les équipes de Weinberg [26] et (opeland [27] ont étudié la prédisposition à développer des tumeurs che\% des souris mutantes pour l'homologue murin du gène de la neurofibromine. les souris homozygotes pour la mulation meurent a mi-gestation (13 jours). Une des anomalies observées che\% ces souris homorygotes est une anomalie du développement du corur entraînant de sévères anomalies cardiaques ainsi qu'une hyperplasie des ganglions sympathiques dérivés de la crête

neurale. I es hétérozygotes ne présentent, quant à eux, aucun signe particulier caractéristique de la neurofibromatose, en dehors d'une prédisposition à différentes proliférations malignes néoplasiques très variées; on peul observer des phéochromocytomes et des leucémies myéloïdes pour les tumeurs associées à une NFI, mais aussi d'autres types de tumeurs comme des leucémies lymphoïdes, des adénocarcinomes du poumon, des hépatomes el des fibrosarcomes. Aucun gliome optique n'a été observé chez ces souris. Une perte de l'allèle non muté est détectéc dans environ $50 \%$ des tumeurs développées par ces souris. I.'ARN est synthé$\mathrm{m} / \mathrm{s} n^{\circ} \mathrm{l}$, vol. $I 1$, junvier 95 tisé à partir des deux allèles che\% la souris hétérozygote. I'ARN correspondant au gène muté code pour une protéine instable entrainant une diminution du laux de la neurofibromine chez les hétérozygotes et une absence totale ou non détectable de la protéine chez les homozygotes.

I. même type de modèle a été développé pour le gène de la p53. Les souris homozygotes pour la mutation ont un développement normal mais sont prédiposées à développer des tumeurs de type lymphome trés précocement. I.es souris hétérozygotes développent des ostéosarcomes et des sarcomes des tissus mous, mais beaucoup plus tardivement. Bien que NFI et p53 soient des gènes suppresseurs de tumeurs, ce dernier semble jouer un rôle moins important dans le développement précoce de l'embryon puisque les mutations homorygotes n'entrainent pas d'anomalic du développement en dehors de cette prédisposition accrue à développer des tumeurs [28].

\section{Application au diagnostic}

Le diagnostic de la neurofibromatose est clinique dans de nombreux cas. Cependant, depuis le clonage du gène et des marqueurs polymorphes flanquants, la biologie moléculaire est utilisée pour confirmer un diagnostic clinique incertain et établir ainsi un diagnostic présymptomatique. Dans les cas familiaux, la stratégie de diagnostic indirect repose sur l'analyse de polymorphisme intragénique ou flanquant. Actuellement, avec le nombre de marqueurs dont nous disposons, au moins $85 \%$ des familles sont informatives. Un fort déséquilibre de liaison a été mis en évidence à l'intérieur du gène pour sept polymorphismes répartis sur $340 \mathrm{~kb}$. Cependant, ce déséquilibre chute considérablement pour le dernier polymorphisme situé en 3' qui se trouve à seulement $68 \mathrm{~kb}$ du précédent, entraînant un pourcentage de recombinaison non négligeable dont on doit tenir compte dans l'établissement du diagnostic. Dans les cas sporadiques, trois approches sont possibles: (1) la recherche de mutations ponctuelles ou de remaniements avec les diffé- rentes techniques disponibles ; (2) l'étude de l'ARN messager directement au niveau des fibroblastes de patients ; (3) l'étude de la séquence peptidique correspondant à différentes régions de l'ARNm qui sont traduites. On commait le très faible rendement de la premiere technique (moins de $10 \%$ de mutations détectées); de plus, l'existence d'un pseudogène sur le chromosome 15 rend difficile la recherche de potentiels de remanicments géniques par cartographic de restriction utilisant l'AIONc comme sonde ; la deuxième ne permettra pas de détecter les mutations touchant la région promotrice et qui aboliraient la transcription clu gène; quant à la troisieme technique, elle permet de comparer les profils de migration de ces peptides dérivés d'un malade atteint de NFI et d'un témoin. Si un profil de migration anormal apparait, on essaie alors de mettre en évidence la mutation au niveau génomique pour la région du gène correspondant au peptide testé. Cette dernière technique semble tres prometteuse.

\section{Conclusion}

L'exploitation des modèles animaux récemment construits devrait permettre de mieux comprendre le rôle de la neurofibromine dans la régulation de $p 21^{\text {kis. }}$, et ainsi de mieux cerner les conséquences de la mutation de ce gène selon le type cellulaire dans lequel il s'exprime. Il est intéressant de noter que dans la sclérose tubéreuse de Bourneville, une autre phacomatose, un gène ayant une homologie avec une autre protéine (;AP est aussi impliqué. I.es conséquences phénotypiques des différentes mutattions dans ces modiles domneront certainement des indications sur de potentiels domaines fonctionnels de la protéine, différents du domaine (;AP. Toutes ces informations permettront de mieux connaître le gène et le fonctionnement de la protéine et apporteront des éléments essentiels à la commaissance des phacomatoses en général, mais surtout de la NFI avec tous les débouchés envisageables en matière de diagnostic moléculaire et clinique et de thérapie génique. 


\section{* LEXIQUE *}

Phacomatose : nom donné à un groupe de maladies qui ont en commun la présence de petites tumeurs ou de kystes situés en divers points du corps, en particulier au niveau du système nerveux. Les plus caractéristiques sont les neurofibromatoses de type I et II, la sclérose tubéreuse de Bourneville, la maladie de von Hippel-Lindau.

Hamartome : nom donné à une malformation, d'aspect tumoral, due ì un mélange anormal des éléments constitutifs normaux.

Phéochromocytome : tumeur très riche en adrénaline et en noradrénaline, développée aux dépens des cellules chromasfines du tissu médullaire de la glande surrénale.

Syndrome de Noonan : syndrome caractérisé par un élargissement anormal de l'espace interoculaire (d'origine congénitale ou acquise), des malformations cardiaques, une débilité mentale et quelquefois des déformations thoraciques.

Syndrome de Watson : syndrome associant une sténose pulmonaire, des troubles conductifs intracardiaques, une dysmorphie crâno-faciale, une débilité mentale et des taches cutanées café au lait.

\section{RÉFÉRENCES}

1. Riccardi VC. Medical progress : von Recklinghausen neurofibromatosis. $N$ Engl J Med $1981 ; 305: 1617-27$

2. Mc Kusick VA. Mendelian inheritance in man. Tenth edition, Baltimore and London : John Hopkins University Press, 1992 :

3. Jadayel D, Fain P, Upadhyaya M, et al. Paternal origin of new mutations in von Reck linghausen neurofibromatosis. Nature $1990 ; 343$ : 558-9.

4. Zlotogora J. Mutations in von Recklinghausen neurofibromatosis : an hypothesis. Am J Med Genet $1993 ; 46: 182-4$.

5. Schmidt MA, Michels VV, Dewald GW Cases of neurofibromatosis with rearrangements of 17 involving band $17 \mathrm{q} 11.2 . \mathrm{Am}$ ] Med Genet 1987 ; 28 : 771-7.

6. Cawthon RM, Weiss R, Xu G, et al. A ma- gene : cDNA sequence, genomic structure, and point mutation. Cell $1990 ; 62: 193$ 201.

7. Marchuk DA, Saulino AM, Tavakkol R, et al. cDNA cloning of the type 1 neurofibromatosis gene : complete sequence of the NFl gene product. Cenomics $1991 ; 11$ :93140

8. Gutmann DH, Andersen I.B, Cole JL, Swaroop M, Collins FS. An alternatively-spliced mRNA in the carboxy terminus of the neurofibromatosis type 1 (NF1) gene is expressed in muscle. Hum Mol Genet 1993 ; 2 : 989-92.

9. Xu G, O'Connell P, Viskochil D, et al The neurofibromatosis type 1 gene encodes a protein related to GAP. Cell 1990; 62 : 599-608.

10. Gutmann DH, Boguski M, Marchuk D, Wigler M, Collins FS, Ballester R. Analysis of the neurofibromatosis type 1 (NF1) GAP-related domain by site-directed mutagenesis. Oncogene $1993 ; 8: 761-9$.

11. White $R$, O'Connell $P$. Identification and characterization of the gene for neurofibromatosis type 1. Curr Op Genet Dev 1991; $1: 15-9$.

12.Upadhyaya $M$, Shen $M$, Cherryson $A$, et al. Analysis of mutations at the neurofibromatosis 1 (NFI) locus. Hum Mol Genet $1992 ; 9: 735-40$.

13. Kayes L.M, Burke W, Riccardi VM, Bennet R, Ehrlich P, Rubenstein A, Stephens K. Deletions spanning the neurofibromatosis I gene : identification and phenotype of five patients. Am J Hum Genet 1994 ; 54 : 424-36.

14. Tassabehji M, Strachan T, Sharland M, et al. Tandem duplication within a neurofibromatosis type 1 (NF1) gene exon in a family with features of Watson syndrome and Noonan syndrome. Am / Hum Genet 1993 ; $53: 90-5$.

15. Easton DF, Ponder MA, Huson SM, Pon$\operatorname{der} \mathrm{AJ}$. An analysis of variation in expression of neurofibromatosis (NF) type 1 (NF1) : evidence for modifying genes. $A m J$ Hum Genel 1993 ; 53 : 305-13.

16. Bonaïti-Pellié C, Feingold J. Rétinoblastome : de l'épidémiologie génétique à la biologie moléculaire. médecine/sciences 1990 ; $6: 972-9$.

17. Xu W, Mulligan I,M, Ponder MA, el al. I coss of $\mathrm{NFI}$ alleles in phaeochromocytomas from patients with type 1 neurofibromatosis. Genes Chromosome Cancer 1992; $4: 337$ 42 .

18. Menon AG, Anderson KM, Riccardi VM el al. Chromosome $17 \mathrm{p}$ deletions and p53 gene mutations associated with the formation of malignant neurofibrosarcomas in von Recklinghausen neurofibromatosi Proc Natl Acad Sci USA 1990; 87 : 5435-9.

19. Legius E, Marchuk DA, Collins FS, Glover TW. Somatic deletion of the neurofibromatosis type 1 gene in a neurofibrosarcoma supports a tumour suppressor gene hypothesis. Nature Genet 1993; 3:122-6.
20. Shannon KM, O'Connell P, Martin GA, Paderanga D, Olson K, Dinndorf P, McCormick $\mathrm{F}$. Ooss of the normal $N F I$ allele from the bone marrow of children with type 1 neurofibromatosis and malignant myeloid disorders. N Engl J Med 1994 ; 330 : 597-601.

21. The I, Murthy AE, Hannigan GE, et al. Neurofibromatosis type 1 gene mutations in neuroblastoma. Nature Genet $1993 ; 3: 62-6$

22. Bader SA, Fasching C, Brodeur GM, Stanbridge E. Dissociation of suppression of tumorigenicity and differentiation in vitro effected by transfer of single human chromosomes into human neuroblastoma cells. Cell Grotuth Diff 1991 ; 2 : 245-55.

23. De Clue JE, Papageorge AG, Fletcher A, et al. Abnormal regulation of mamma\{ian p21-ras contributes to malignant tumor growth in von Recklinghausen (type 1) neurofibromatosis. Cell $1992 ; 69$ : 265-73.

24. Junien C, Henry I. The genetics of Wilms' tumor: a blend of aberrant development and genomic imprinting. Kidney Int $1994 ; 46$ : 264-79.

25. Hoffmeyer S, Assum G, Kaufmann, Krone $\mathrm{W}$. Unequal expression of $N F 1$ alleles. Nalure Genet 1994;6:331.

26. Jacks $T$, Shih $S$, Schmitt EM Bronson RT, Bernards A, Weinberg RA. Tumour predisposition in mice heterozygous for a targeted mutation in NFl. Nature genel $1994 ; 7: 353-61$.

27. Brannan CI, Perkins AS, Vogel KS, et al. Targeted disruption of the neurofibromato sis type-1 gene leads to developmental abnormalities in heart and various neural crest-derived tissues. Genes Dev 1994:8. 1019-29.

28. Harvey M, McArthur MJ, Montgomery CA, Butel IS, Bradley A, Donehower IA Spontaneous and carcinogen-induced tumorigenesis in p53-deficient mice. Nature Genel $1993 ; 5$ : 225-9.

\section{Isabelle Henry}

Inserm U. 383, hôpital Necker, clinique Maurice-lamy, 149, rue de Sèvres, 75015 Paris, France.

\section{TIRÉS À PART}

1. Henry. 